\title{
A novel five-phase fault-tolerant modular in-wheel permanent-magnet synchronous machine for electric vehicles
}

\author{
Yi Sui, ${ }^{1}$ Ping Zheng, ${ }^{1, a)}$ Fan Wu, ${ }^{1}$ Pengfei Wang, ${ }^{1}$ Luming Cheng, ${ }^{1}$ and Jianguo Zhu ${ }^{2}$ \\ ${ }^{1}$ School of Electrical Engineering and Automation, Harbin Institute of Technology, Harbin 150080, China \\ ${ }^{2}$ Center for Electrical Machines and Power Electronics, University of Technology Sydney, Sydney, NSW2007, \\ Australia
}

(Presented 6 November 2014; received 22 September 2014; accepted 9 November 2014; published online 30 March 2015)

\begin{abstract}
This paper describes a five-phase fault-tolerant modular in-wheel permanent-magnet synchronous machine (PMSM) for electric vehicles. By adopting both the analytical and finite-element methods, the magnetic isolation abilities of some typical slot/pole combinations are analyzed, and a new fractional-slot concentrated winding topology that features hybrid single/double-layer concentrated windings and modular stator structure is developed. For the proposed hybrid single/double-layer concentrated windings, feasible slot/pole combinations are studied for three-, four-, and five-phase PMSMs. A five-phase in-wheel PMSM that adopts the proposed winding topology is designed and compared with the conventional PMSM, and the proposed machine shows advantages of large output torque, zero mutual inductances, low short-circuit current, and high magnetic isolation ability. Some of the analysis results are verified by experiments. ( 2015 AIP Publishing LLC.

[http://dx.doi.org/10.1063/1.4915477]
\end{abstract}

With the increasing aggravation of energy crisis and air pollution, electric vehicles (EVs) have drawn wide interests in recent years. Owing to the elimination of mechanical gearboxes, in-wheel direct-drive system enables higher system efficiency and better control of EVs. ${ }^{1,2}$ Since the in-wheel electric drive system is the only power source for pure EVs, so continuous operation after faults is very important. Multiphase permanent-magnet (PM) machine that features advantages of high power density and high reliability is promising for in-wheel direct-drive systems. ${ }^{3-6}$

Fractional-slot concentrated windings (FSCWs) are widely adopted for fault-tolerant PM synchronous machines (PMSMs). However, FSCW introduces many space harmonics in the air gap, which takes negative effects on machine performance. The impacts of slot/pole combinations on eddycurrent losses of PMs are analyzed, and single-layer FSCW leads to more rotor losses than double-layer FSCW. ${ }^{7,8}$ For the double-layer FSCW, its magnetic isolation ability is poorer than that of the single-layer FSCW.

Recently, modular-structure machines are increasingly investigated due to their characteristics of increased faulttolerant ability and simplified manufacturing process. ${ }^{9,10}$ For multiphase fault-tolerant PM machines, if the phase failure involves only a part of the machine, the fault part can be replaced by a healthy module, which leads to easy maintenance. Three-phase modular PM machine that features single-layer FSCW is reported. ${ }^{11}$ Herein, we report a novel five-phase fault-tolerant PMSM, characterized as hybrid single/double-layer windings, modular stator structure, and high magnetic isolation.

Five-phase PMSMs with FSCWs have been developed. ${ }^{12}$ The investigated machines include a 20-slot/18-pole PMSM

${ }^{\text {a)} E l e c t r o n i c ~ m a i l: ~ z h e n g p i n g @ h i t . e d u . c n . ~}$ with single-layer FSCW, a 20-slot/18-pole PMSM with double-layer FSCW, and a 30-slot/28-pole PMSM with double-layer FSCW, as shown in Fig. 1. The magnetic isolation ability is investigated by finite-element method (FEM). With PMs removed and only one phase winding activated, the flux distributions are calculated by FEM, as shown in Fig. 2 .

For the 20-slot/18-pole single-layer winding configuration, alternate teeth are wound, and the non-wound teeth are named fault-tolerant teeth. The flux linkage in the fault-tolerant teeth is slot leakage flux, and the flux activated by Phase A does not link the other four phases, so there is no magnetic coupling between different phases. For the 20-slot/18-pole double-layer FSCW, all teeth are wound, no flux linkage that crosses the air gap links the other phases, and only a little slot leakage flux links the adjacent phases. For the 30-slot/28-pole double-layer FSCW, the flux activated by Phase A links the other four phases, and the coupling flux linkage includes both the slot leakage flux and the flux linkage that crosses the air gap. Among the three winding configurations, the 30-slot/28-pole double-layer FSCW has the lowest magnetic isolation ability.

The mutual inductance of stator phase windings can be calculated by using the corresponding winding functions. The mutual inductance is given by $L_{a b}=$ $\frac{\mu_{0} r l}{g} \int_{0}^{2 \pi} N_{a}(\varphi) N_{b}(\varphi) d \varphi$, where $r$ is the diameter of air gap, $l$ is the efficient length of stator, and $g$ is the length of air gap.

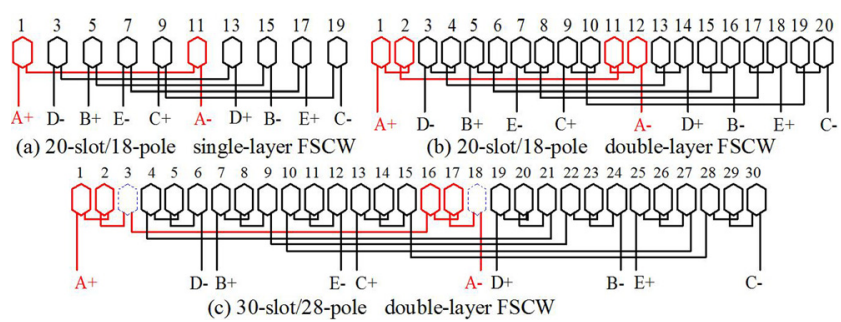

FIG. 1. Winding configurations of the five-phase PMSMs. 


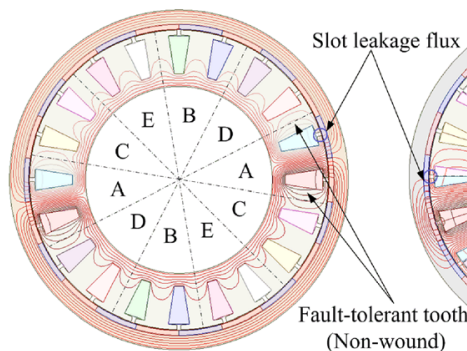

(a)

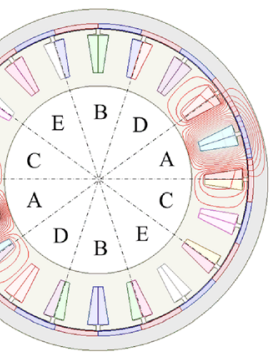

(b)

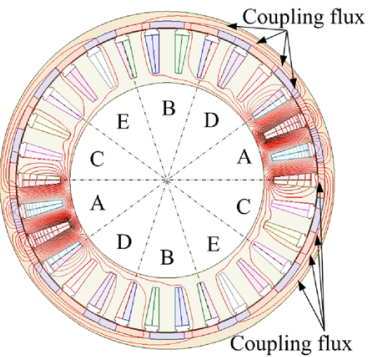

(c)
FIG. 2. Flux distributions (only Phase A activated): (a) 20-slot/18-pole single-layer FSCW, (b) 20-slot/18-pole double-layer FSCW, and (c) 30-slot/ 28-pole double-layer FSCW.
For the aforementioned three winding configurations, the winding functions of Phase A are shown in Fig. 3 (the winding functions in Fig. 3 are corresponding to the coils marked with red solid and blue dashed lines in Fig. 1, respectively). The winding functions of the other four phases are similar to that of Phase A with phase shift of $2 \pi / 5$.

Compared with both the 20-slot/18-pole single- and double-layer windings, the winding function of 30-slot/28pole features nonzero values within the coordinate range of $[-\pi,-0.6 \pi],[-0.4 \pi, 0.4 \pi]$, and $[0.6 \pi, \pi]$, which results in nonzero values of mutual inductance, i.e., magnetic coupling between different phase windings. Hence, if the winding function marked with blue dashed lines in Fig. 3(c) is removed, which means coils 3 and 18 in Fig. 1(c) are removed from Phase A, the mutual inductance should be zero. Similar operations are executed for the other four phases, and a novel winding topology that characterizes as hybrid single/double-layer windings is obtained, as shown in Fig. 4.

After the coils are removed from the conventional 30-slot/ 28-pole PMSM, the slot size has to be readjusted. The removed turns should be added to the coils remaining in the slots, so the slot size should be increased, and the slot which contains only one coil side should be decreased. Correspondingly, the dimensions of stator teeth are also adjusted. Stators of both the conventional and proposed five-phase PMSMs are shown in Fig. 5. Each stator module contains three slots, two armature teeth, and two fault-tolerant teeth. The two coils wound on the armature teeth are series-opposing connected. The unwound faulttolerant teeth operate as parts of the magnetic path of slot leakage flux, and the fault-tolerant teeth provide physical,

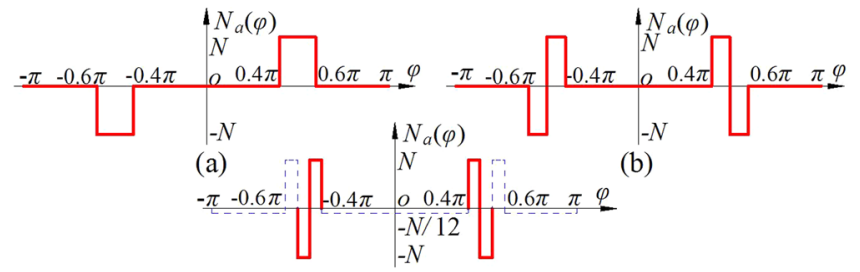

(c)

FIG. 3. Winding functions of Phase A: (a) 20-slot/18-pole single-layer FSCW, (b) 20-slot/18-pole double-layer FSCW, (c) 30-slot/28-pole doublelayer FSCW.

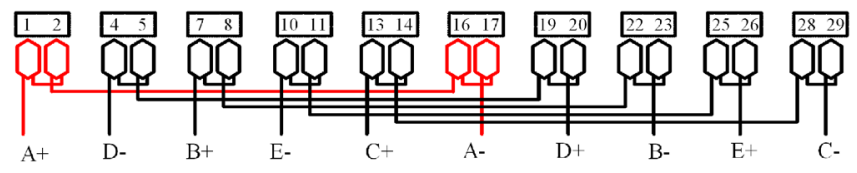

FIG. 4. The proposed hybrid single/double-layer winding configuration. magnetic, electrical, and thermal isolation for different phase windings. Owing to the high magnetic isolation ability, the machine can be assembled by modular stator modules. Besides, in order to link more flux, the coil span can be increased by changing the width of armature tooth.

There are many feasible slot/pole combinations for the proposed hybrid single/double-layer winding configuration. Aiming to maximize the winding factor, the number of stator slots $(Q)$ and the number of rotor poles $(2 p)$ should differ by the smallest possible integer. For the proposed winding topology, each stator module contains 3 slots, so $Q$ must be divisible by 15 for a five-phase machine, and $Q$ and $2 p$ should satisfy $Q=15 n=2 p \pm 1$ or $2 p \pm 2$, where $n=1,2,3 \ldots$ is a positive integer. For a five-phase winding, the phase shift between different phases should satisfy $\theta=72 p$ the phase shift $\theta$ should equal either $\pm 360 k+72$ or $\pm 360 k+144$, where $k=0,1,2,3 \ldots$ is a positive integer. Slot/pole combinations are derived, as listed in Table I.

The slot/pole combinations marked with underlines are multi-duplications of the basic slot/pole combinations. For example, the 45-slot/42-pole scheme is a triple-duplication of the 15-slot/14-pole scheme. Feasible slot/pole combinations for three- and four-phase PMSMs can be derived by using $Q=9 n=2 p \pm 1$ (or $2 p \pm 2$ ), and $Q=12 n=2 p \pm 1$ (or $2 p \pm 2$ ), respectively.

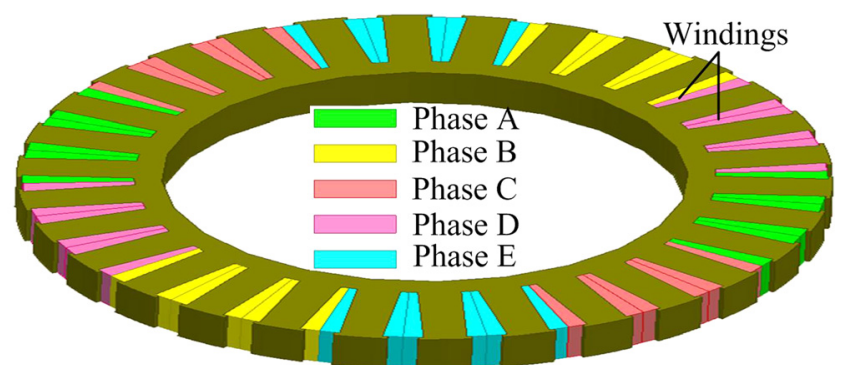

(a) conventional stator (all teeth wound)

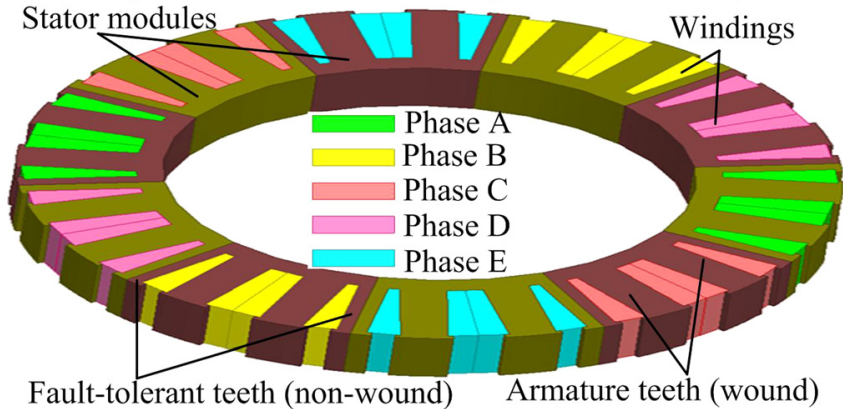

(b) modular stator

FIG. 5. The conventional and proposed modular stators. 
TABLE I. Feasible slot/pole combinations for five-phase modular PMSM.

\begin{tabular}{lc}
\hline \hline Number of slots, $Q$ & Number of pole-pairs, $p$ \\
\hline 15 & 7,8 \\
30 & 14,16 \\
45 & $\underline{21}, 22,23, \frac{24}{28}, 29,31, \underline{32}$ \\
60 & $\cdots$ \\
$\cdots$ & \\
\hline \hline
\end{tabular}

Two five-phase 30-slot/28-pole PMSMs which adopt the conventional and proposed winding configurations are optimally designed under the same volumetric constraint. For the machine which adopts the proposed hybrid single/double-layer winding configuration, the widths of the armature teeth are optimized to maximize the coil flux linkage. The two machines are compared in the following text.

For the two machines, the no-load air-gap flux densities are calculated, as shown in Fig. 6. The dominant harmonic of no-load air-gap flux density is the 14th-order working harmonic. Due to the modular stator structure, no-load air-gap flux density of the proposed machine contains more harmonics than that of conventional machine.

With PMs removed, the air-gap flux densities produced by armature windings are calculated, as shown in Fig. 7. For the conventional machine, the air-gap flux densities contain harmonics with orders of $4,5,14,16,44,46$, etc., and the 14 th-order harmonic is the working harmonic. For the proposed machine, the air-gap flux densities contain more harmonics. The dominant harmonics for the proposed machine are 4th, 6th, 14th, and 16th order harmonics, and the 14thorder harmonic interacts with the 14th-order PM field to generate electromagnetic torque.

Cogging torque is generated due to the interaction between slotted stator and PMs. The frequency of cogging torque in one mechanical rotation is: $f_{\text {cogging }}=\operatorname{LCM}(Q, 2 p)$, where $L C M$ is the least common multiple of the number of slots and poles. Cogging torque waveforms of the two machines are calculated, as shown in Fig. 8.

For the conventional machine, the frequency of cogging torque equals the $L C M$ of 30 and 28, i.e., 420 . For the proposed machine, its stator is assembled by 10 stator modules, so the frequency of its cogging torque equals the $L C M$ of 10 and 28 , i.e., 140 . Since the cogging torque frequency of the proposed machine is lower than that of the conventional machine, so the amplitude of its cogging torque is larger.

For the two machines, both the back electromotive force (emf) waveforms and electromagnetic torque are calculated, as shown in Fig. 9. Compared with the conventional machine, the proposed machine exhibits larger amplitudes of

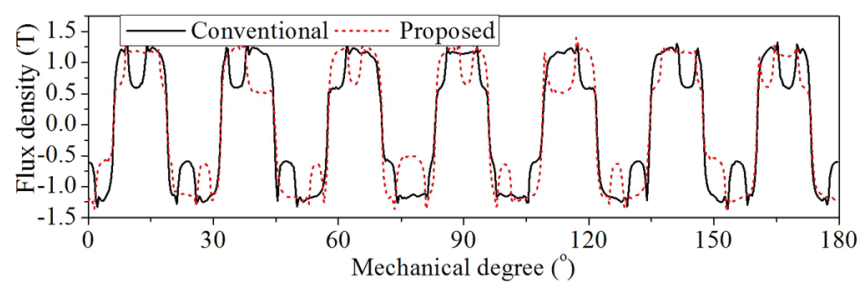

FIG. 6. No-load air-gap flux density.

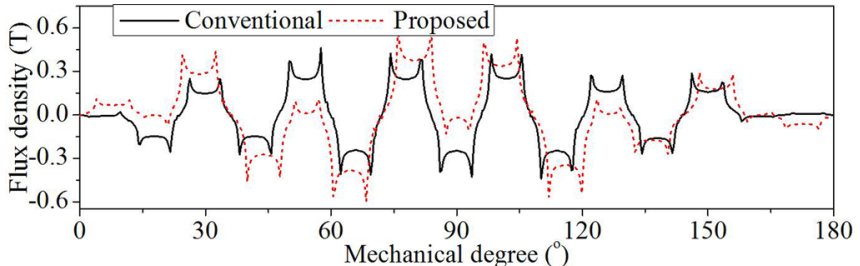

(a) air-gap flux density

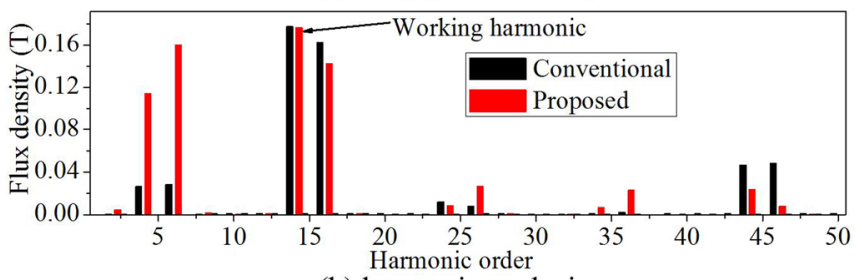

(b) harmonic analysis

FIG. 7. Air-gap flux densities (produced by armature windings).

both the fundamental and 3rd-order harmonic of back emf. Furthermore, the proposed machine outputs larger torque than the conventional machine. Due to the larger amplitude of cogging torque, torque ripple of the proposed machine is larger than that of the conventional machine.

With PMs removed and only one phase winding activated, the flux distributions of the two machines are calculated to compare the magnetic isolation ability of the two machines. The flux distribution of the conventional machine is shown in Fig. 2(c), and flux distribution of the proposed machine is shown in Fig. 10.

The fault-tolerant teeth of the proposed machine isolate Phase A from other phases, and the flux linkage due to Phase A does not link the other phases. While for the conventional machine, the flux linkage due to Phase A links the other

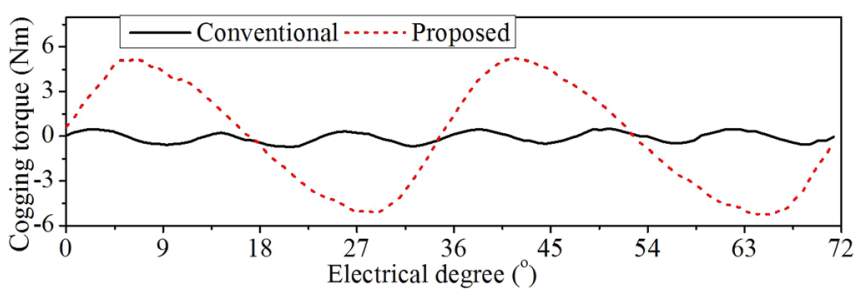

FIG. 8. Cogging torque.

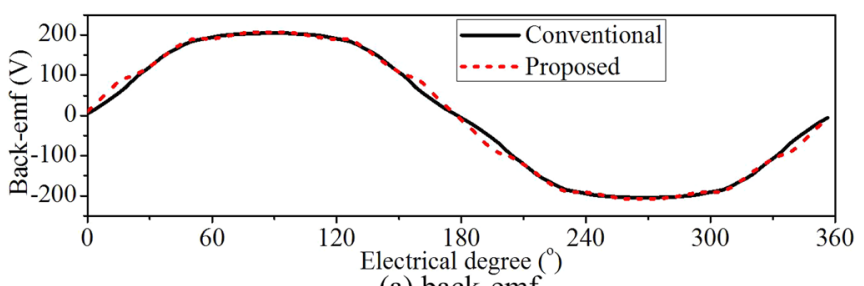

(a) back-emf

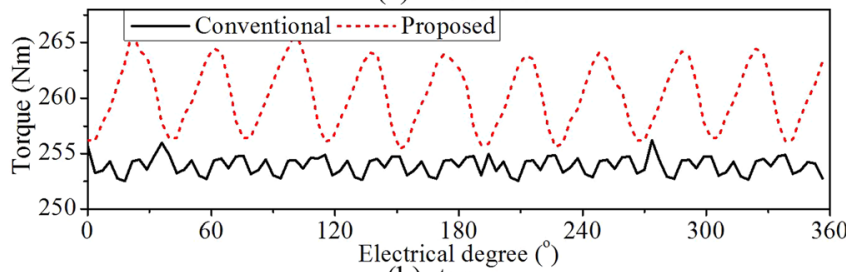

(b) torque

FIG. 9. Back-emf and torque. 


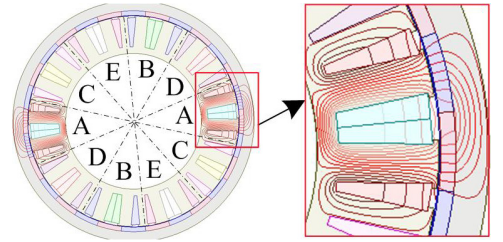

FIG. 10. Flux distribution of the proposed machine (only Phase A activated).

TABLE II. Inductances comparison.

\begin{tabular}{lcclll}
\hline \hline Winding layouts & $L_{A A}(\mathrm{mH})$ & $M_{A B}(\mathrm{mH})$ & $M_{A C}(\mathrm{mH})$ & $M_{A D}(\mathrm{mH})$ & $M_{A E}(\mathrm{mH})$ \\
\hline Conventional & 8.121 & 0.086 & 0.51 & 0.51 & 0.086 \\
Proposed & 10.607 & 0 & 0 & 0 & 0 \\
\hline \hline
\end{tabular}

phases. Self and mutual inductances of both the conventional and proposed machines are calculated, as listed in Table II.

$L_{A A}$ is self-inductance of Phase $A, M_{A B}, M_{A C}, M_{A D}$, and $M_{A E}$ are mutual inductances between Phase $A$ and Phases $B$, $C, D$, and $E$, respectively. For the proposed machine, its selfinductance is larger than that of the conventional machine, and the mutual inductances of the proposed machine are zero, which indicates good magnetic isolation ability. As faults happen, the phase failure can be isolated without affecting the other healthy phases.

The investigated fault-tolerant machines could operate under fault conditions such as open circuit or short circuit of phase windings. Under open-circuit conditions, the fault phase has no effect on the other healthy phases, and the machine performance can be improved by adopting proper fault-tolerant control strategies. ${ }^{12-14}$ However, under shortcircuit condition, current is induced in the fault phase windings due to the existence of PMs, and the induced shortcircuit current affects other healthy phases. For both the conventional and proposed machines, both short-circuit current and back emf are calculated, as shown in Fig. 11.

The phase short-circuit current of the conventional machine is larger than that of the proposed machine. For the conventional machine, the back emf waveforms become asymmetric in short-circuit condition, the back emf of Phases $C$ and $D$ is lower than that of Phases $B$ and $E$, and this is due to the relatively larger mutual inductances between Phase $A$ and Phases $C$ and $D$. Owing to the good magnetic isolation ability of the hybrid single/double-layer winding configuration, shortcircuit phase of the proposed machine has no effect on the other healthy phases, which can be reflected from the symmetric back emf waveforms under short-circuit condition. Hence, short-circuit phase of the proposed machine is effectively isolated from the other healthy phases, and further fault-tolerant control strategies can be developed to deal with the shortcircuit fault, which is not the research point of this paper.

Since PM rotors of the conventional and proposed machines are the same, only one rotor was manufactured to reduce the cost. So far the prototype which adopts the conventional double-layer winding topology was assembled and tested, and the proposed machine is still under construction. The back emf and inductances of the conventional machine are tested. The measured self and mutual inductances are $8.905 \mathrm{mH}, \quad 0.089 \mathrm{mH}, \quad 0.504 \mathrm{mH}, \quad 0.46 \mathrm{mH}, \quad 0.068 \mathrm{mH}$,

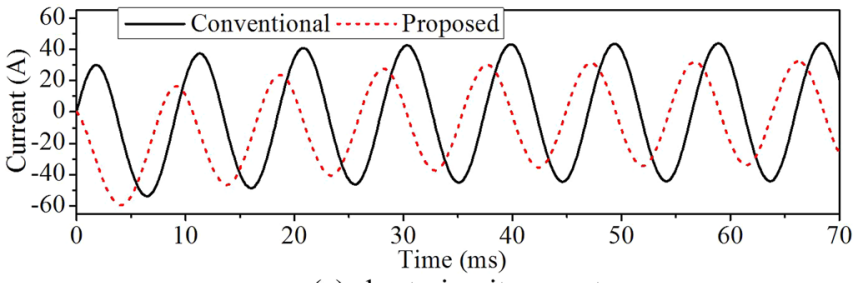

(a) short-circuit current

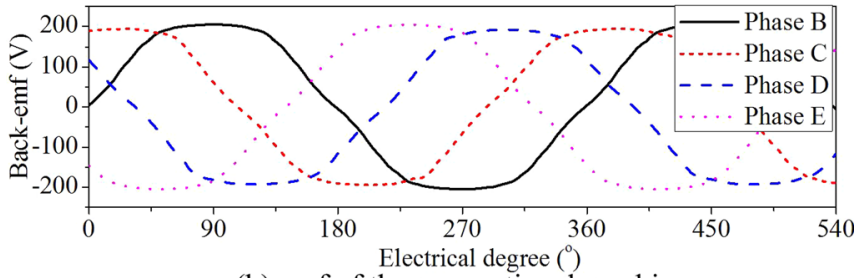

(b) emf of the conventional machine

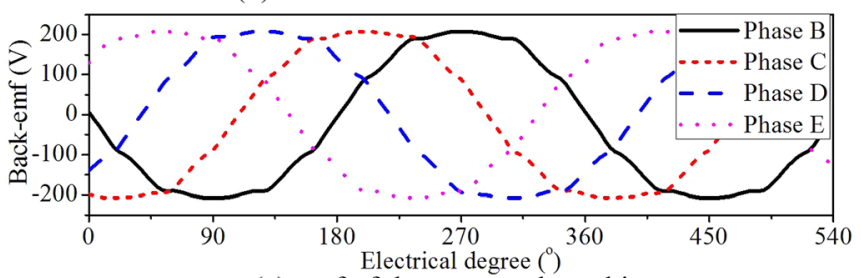

(c) emf of the proposed machine

FIG. 11. Short-circuit current and back-emf under short-circuit condition.

respectively. The test results agree well with the FEM calculated ones, so the FEM calculated results are reasonable.

A novel five-phase fault-tolerant PMSM, which characterizes as hybrid single/double-layer windings and modular stator structure, has been proposed for EVs. Feasible slot/pole combinations of the proposed winding topology have been studied. In comparison with the conventional PM machine, the proposed machine features advantages of high output torque, zero mutual inductances, low short-circuit current, and high magnetic isolation between different phases, but its cogging torque is larger, and the armature reaction field contains more harmonics. Some of the analysis results are verified by experiments. The proposed topology is applicable to arbitrary phase PM machines, and this investigation opens up exploration of modular concept design that features hybrid single/doublelayer windings, especially in multiphase machine fields.

This work was supported by National Natural Science Foundation of China under Project Nos. 51325701 and 51377033.

${ }^{1}$ A. J. Rix et al., IEEE Trans. Ind. Electron. 59, 2475 (2012).

${ }^{2}$ I. S. Suh et al., in IEEE International Electric Machines and Drives Conference (IEMDC), Chicago, USA, 12-15 May 2013 (IEEE, 2013), pp. $1235-1240$.

${ }^{3}$ A. M. El-Refaie, IET Electr. Power Appl. 5, 59 (2011).

${ }^{4}$ M. G. Simoes et al., IEEE Trans. Ind. Electron. 49, 1154-1164 (2002).

${ }^{5}$ C. J. Ifedi et al., IEEE Trans. Ind. Appl. 49, 1249 (2013).

${ }^{6} \mathrm{G}$. Liu et al., J. Appl. Phys. 111, $07 \mathrm{E} 713$ (2012).

${ }^{7}$ B. Aslan et al., IEEE Trans. Energy Convers. 29, 72 (2014).

${ }^{8}$ G. J. Atkinson et al., IEEE Trans. Ind. Appl. 42, 1162 (2006).

${ }^{9} \mathrm{G}$. Li et al., IEEE Trans. Energy Convers. 29, 716 (2014).

${ }^{10}$ J. Wang et al., IEEE Trans. Veh. Technol. 54, 809 (2005).

${ }^{11}$ E. Fornasiero et al., IEEE Trans. Ind. Appl. 49, 1316 (2013).

${ }^{12} \mathrm{~N}$. Bianchi et al., IEEE Trans. Ind. Electron. 55, 1978 (2008).

${ }^{13}$ L. Parsa et al., IEEE Trans. Veh. Technol. 56, 1546 (2007).

${ }^{14}$ S. Dwari et al., IEEE Trans. Ind. Electron. 58, 476 (2011). 